\title{
Ciudad Real y Puertollano. Áreas funcionales urbanas (FUA) y policentrismo en Castilla-La Mancha (España)
}

\author{
María del Carmen Cañizares Ruiz \\ Héctor Samuel Martínez Sánchez-Mateos \\ Universidad de Castilla-La Mancha. Departamento de Geografía y Ordenación del Territorio \\ MCarmen.Canizares@uclm.es \\ HectorS.Martinez@uclm.es
}

Recepción: noviembre de 2012

Aceptación: enero de 2013

\begin{abstract}
Resumen
Las áreas funcionales urbanas (FUA) de Ciudad Real y de Puertollano se estructuran a partir de ambas ciudades, funcionalmente diferentes pero que sostienen unas relaciones cada vez más intensas en el sector suroccidental de la región de Castilla-La Mancha, dentro de la provincia de Ciudad Real. Las interrelaciones se establecen en base a las infraestructuras de comunicación, principalmente la línea de alta velocidad ferroviaria. Este artículo caracteriza ambas áreas funcionales y determina las relaciones entre sus núcleos principales en base a una metodología que combina análisis estadístico (población, crecimiento real anual, población vinculada, etc.) y empírico, con las representaciones cartográficas de los principales indicadores. Los resultados obtenidos permiten abordar una caracterización conjunta de ambas áreas funcionales en el contexto del policentrismo aplicado a la región de Castilla-La Mancha y del importante papel articulador que las infraestructuras de alta capacidad tienen en este caso de estudio.
\end{abstract}

Palabras clave: sistemas urbanos regionales; policentrismo; área funcional urbana; Ciudad Real; Puertollano; Castilla-La Mancha.

Resum. Ciudad Real i Puertollano. Àrees funcionals urbanes (FUA) i policentrisme a Castellala Manxa (Espanya)

Les àrees funcionals urbanes (FUA) de Ciudad Real i de Puertollano s'estructuren entorn de totes dues ciutats, funcionalment diferents, però que mantenen relacions cada cop més intenses en el sector sud-oest de la regió de Castella-la Manxa, a la província de Ciudad Real. Aquestes interrelacions es fonamenten en les infraestructures de comunicació, principalment la línia de ferrocarril d'alta velocitat. El present article caracteritza totes dues àrees funcionals i determina les relacions entre els nuclis principals, fent servir una metodologia que combina l'anàlisi estadística (població, creixement anual real, població vinculada, etc.) i empírica, amb representacions cartogràfiques dels indicadors principals. Els resultats obtinguts permeten abordar una caracterització conjunta de totes dues àrees funcionals en 
el context del policentrisme aplicat a la regió de Castella-la Manxa i de l'important paper articulador que tenen les infraestructures d'alta capacitat en aquest cas d'estudi.

Paraules clau: sistemes urbans regionals; àrea funcional urbana; policentrisme; Puertollano; Ciudad Real; Castella-la Manxa.

Résumé. Ciudad Real et Puertollano. Aires urbaines fonctionnelles (FUA) et polycentrisme à Castilla-La Mancha (Espagne)

Les aires urbaines fonctionnelles (FUA) de Ciudad Real et Puertollano sont localisées dans le secteur sud-ouest de la région du Castilla-La Mancha, dans la province de Ciudad Real. Ces aires sont organisées par deux villes différentes sur le plan fonctionnel, mais qui maintiennent des relations de plus en plus intenses. Ceux-ci sont définis sur les infrastructures de communication, principalement la ligne de chemin de fer à grande vitesse. Le but de cet article est de caractériser les deux domaines fonctionnels et de déterminer les relations entre leurs principaux centres fondées sur une méthode qui combine l'analyse statistique (population, croissance annuelle réelle, population liée, etc.) et empiriques, avec des représentations cartographiques des principaux indicateurs. Les résultats obtenus permettent de conduire une caractérisation conjointe de ces deux aires urbaines fonctionnelles dans le contexte du polycentrisme appliqué à la région de Castilla-La Mancha, Espagne, et du important rôle articulateur des infrastructures de haute capacité dans ce cas d'étude.

Mots clé: systèmes urbains régionaux; polycentrisme; aires urbaines fonctionnelles; Ciudad Real; Puertollano; Castilla-La Mancha.

Abstract. Ciudad Real and Puertollano. Functional Urban Areas (FUA) and polycentrism in Castilla-La Mancha (Spain)

The Functional Urban Areas (FUA) of Ciudad Real and Puertollano are structured by both cities, which are functionally different but keeping growing mutual relations. These areas are located to the south-west of Castilla-La Mancha region, within the province of Ciudad Real. Transport infrastructures, mainly the high-speed rail line, are the main factor that explains the interrelations. The aim of this paper is to characterize both functional areas and to determine the relations between their main urban centres. The methodology combines statistical (population, annual growth rate, linked population, etc.) and empirical analyses, using the cartographic visualization of the main indicators. The results confirm that a joint analysis of both FUA is useful in the context of the polycentrism present in the region of Castilla-La Mancha (Spain) and of the important structuring role of the high capacity transport networks in this case-study.

Keywords: regional urban networks; polycentrism; Functional Urban Areas; Ciudad Real; Puertollano; Castilla-La Mancha.

\section{Sumario}

1. Introducción: Policentrismo y FUA en el marco de la Unión Europea

2. La aplicación del policentrismo a la región de Castilla-La Mancha

3. Las áreas funcionales urbanas (FUA) de Ciudad Real y Puertollano
4. La conexión entre Ciudad Real y Puertollano y sus comunicaciones

5. Conclusiones

Referencias bibliográficas 


\section{Introducción: Policentrismo y FUA en el marco de la Unión Europea}

En la Unión Europea, la componente territorial ha ido alcanzando un protagonismo progresivo desde finales del siglo XX, desde la aprobación de la Estrategia Territorial Europea (1999), hasta el momento actual, caracterizado por la cohesión territorial, en la búsqueda de un desarrollo regional mejor y más justo. En este contexto, como afirmó J. Farinós, la «recuperación del territorio» se debía vincular al giro territorial de las políticas y a la necesidad de planificar correctamente el futuro (Cañizares, 2007: 4). El desarrollo territorial policéntrico y las nuevas relaciones entre el campo y la ciudad se planteaban, entonces, como una de las tres directrices básicas que conducían a la cohesión territorial, junto con el acceso equitativo a las infraestructuras y al conocimiento, así como la gestión prudente de la naturaleza y el patrimonio cultural (UE, 1999).

La consecución de un desarrollo equilibrado, armonioso, sostenible y policéntrico se sustenta, precisamente, en una reorientación de las relaciones entre el campo y la ciudad, así como en una estructuración basada en los centros urbanos de más de 15.000 habitantes, capaces de organizar a su alrededor un área funcional urbana o FUA, que albergará un territorio flexible con población superior a 50.000 habitantes (Aalbu, 2004: 169-170). Así, el policentrismo se ha consolidado como un elemento imprescindible de la ordenación territorial, aún habiendo sido «objeto de múltiples interpretaciones: como herramienta para la planificación; como forma de estructura urbana; como objetivo de la política socioeconómica; y como imagen para el marketing del lugar» (Cañizares, 2007: 5). En cualquier caso, una solución para un desarrollo equilibrado y una mayor cohesión territorial, «ya que, en definitiva, se trata de articular todos los municipios conectándolos a núcleos urbanos rectores» (Pillet, 2012: 36).

La ETE no es un documento ejecutivo, puesto que carece de ciertas especificaciones necesarias a la hora de definir de forma efectiva el policentrismo y su articulación sobre los territorios. Así lo demuestran los trabajos derivados de la red POLYNET, que analizó la estructura policéntrica de 8 aglomeraciones urbanas europeas ${ }^{1}$, en los que se apreciaban problemas a la hora de definir los límites y establecer la funcionalidad de estas estructuras urbanas en red (Hall y Pain, 2006). Ante esta circunstancia, la literatura científica propone dos diferentes concepciones del policentrismo: una de carácter morfológico y relacionada con la estructura de la red urbana y que bebe de la tradición del lugar central, y otra de carácter funcional, de estructura espacial más compleja y asentada en los vínculos de complementariedad (Green, 2007). Ambas visiones no se contraponen, sino que ofrecen ópticas complementarias de un fenómeno relacionado con la reciente evolución de las estructuras urbanas en un escenario de crecientes interrelaciones e interdependencias.

Sin embargo, el interés por desarrollar un modelo territorial equilibrado y funcional no ha sido un elemento clave del discurso político de la mayoría

1. París, Bélgica central, Dublín, norte de Suiza, Ranstad holandés, Rhin principal, Rhin-Rhur y sureste de Inglaterra (www.polynet.org.uk). 
de gobiernos autonómicos del Estado español, donde la visión territorial ha quedado ocultada por la importancia de las políticas sectoriales, o bien porque la comarcalización ha sido vaciada de contenido desde sus inicios, debido a múltiples problemas (complejidad de su definición, peso de la independencia municipal, tradición de las administraciones provinciales o pujanza de las áreas metropolitanas) (Pillet et al., 2007: 647).

Actualmente, en el marco de la Unión Europea, después de la aprobación de la Agenda Territorial (UE, 2007) y, sobre todo, de la Estrategia "Europa 2020» y de la renovada Agenda Territorial Europea 2020 (UE, 2011), la cohesión territorial se presenta como objetivo común para los países miembros de la Unión Europea para la construcción de una Europa «más armoniosa y equilibrada» (UE, 2011) y, en este contexto, el policentrismo sigue siendo una prioridad y puede contribuir a paliar los desequilibrios socioeconómicos y territoriales en la Europa comunitaria, principalmente en la periferia y en la escala subestatal, en relación con las estrategias territoriales de carácter regional. Quizás una de sus claves es su caracterización a diferentes escalas, pero en España se le ha prestado una atención aún limitada, ya que sólo se han estudiado, a partir de diferentes metodologías, las principales metrópolis españolas (Madrid, Barcelona, Valencia, Bilbao, Sevilla, Zaragoza y Málaga), el área metropolitana de Barcelona de manera individualizada y algunas comunidades autónomas, como Madrid y Castilla-La Mancha, según recoge un reciente estudio (Marmolejo, Chica y Masip, 2011: 170).

\section{La aplicación del policentrismo a la región de Castilla-La Mancha}

En el caso español, el modelo territorial definido por la UE (policéntrico, referenciado a escalas de proximidad, etc.) se ha manifiestado, principalmente, a través de las transformaciones experimentadas por el propio Estado y la emergencia de nuevos actores a diferentes escalas vinculados con estrategias de cooperación, promoción económica y desarrollo territorial, así como la consolidación de la democracia, la participación ciudadana, los nuevos valores ambientales y aspectos como la repolitización (Farinós, 2001).

Consideramos que, en los niveles regionales y locales, las estrategias territoriales deben orientarse al diseño de modelos urbanos policéntricos conectados con las zonas rurales adaptados a las características de cada espacio geográfico (Pillet et al., 2007: 627). Y esto, precisamente, es lo que, desde el análisis geográfico, hemos abordado en el Grupo de Investigación DETER (Desarrollo Territorial de Castilla-La Mancha), en el que venimos trabajando, en los últimos años, sobre cómo trasladar la Estrategia Territorial Europea a la región de Castilla-La Mancha (España) (Pillet et al., 2007; Pillet et al., 2010), aplicando el policentrismo en la escala regional o subestatal.

Para la delimitación de centros y subcentros en el territorio regional, así como de los flujos de interrelación, se utilizaron diversas fuentes (Pillet et al., 2007: 633 y s.) y fue especialmente destacable la "población vinculada" extraída del censo de 2001 (y lamentablemente desaparecida en el de 2011), es decir, aquella población no residente en el municipio pero estrechamen- 
te relacionada con él en función de la movilidad de la población por razones laborales, por estudios y por segunda residencia. Información que fue completada con el crecimiento demográfico para el período 2001-2008. Los resultados obtenidos de mayor relevancia permitieron la delimitación de 10 áreas funcionales urbanas o FUA en un territorio con un alto componente de ruralidad y escasamente urbanizado, localizado en el interior de la España peninsular y con una vertebración precaria, siempre muy dependiente de la capital del Estado. Estas FUA, que organizan los municipios de Albacete, Cuenca, Ciudad Real, Puertollano, Talavera de la Reina, Guadalajara, Alcázar de San Juan-Tomelloso, Toledo, Illescas y Valdepeñas, abarcan la totalidad del espacio regional y, por tanto, de su población (2.115.334 hab. en 2011), presentan diferentes dinámicas y se estructuran a partir de 5 áreas dependientes de primer nivel (Villarrobledo-San Clemente, Hellín, Manzanares, Almansa y Tarancón) y 10 de segundo nivel (Ocaña, Quintanar de la Orden, Torrijos, Sonseca, Motilla del Palancar, Villanueva de los Infantes, Almadén, CasasIbáñez, Molina de Aragón y Sigüenza), como podemos observar en la figura 1.

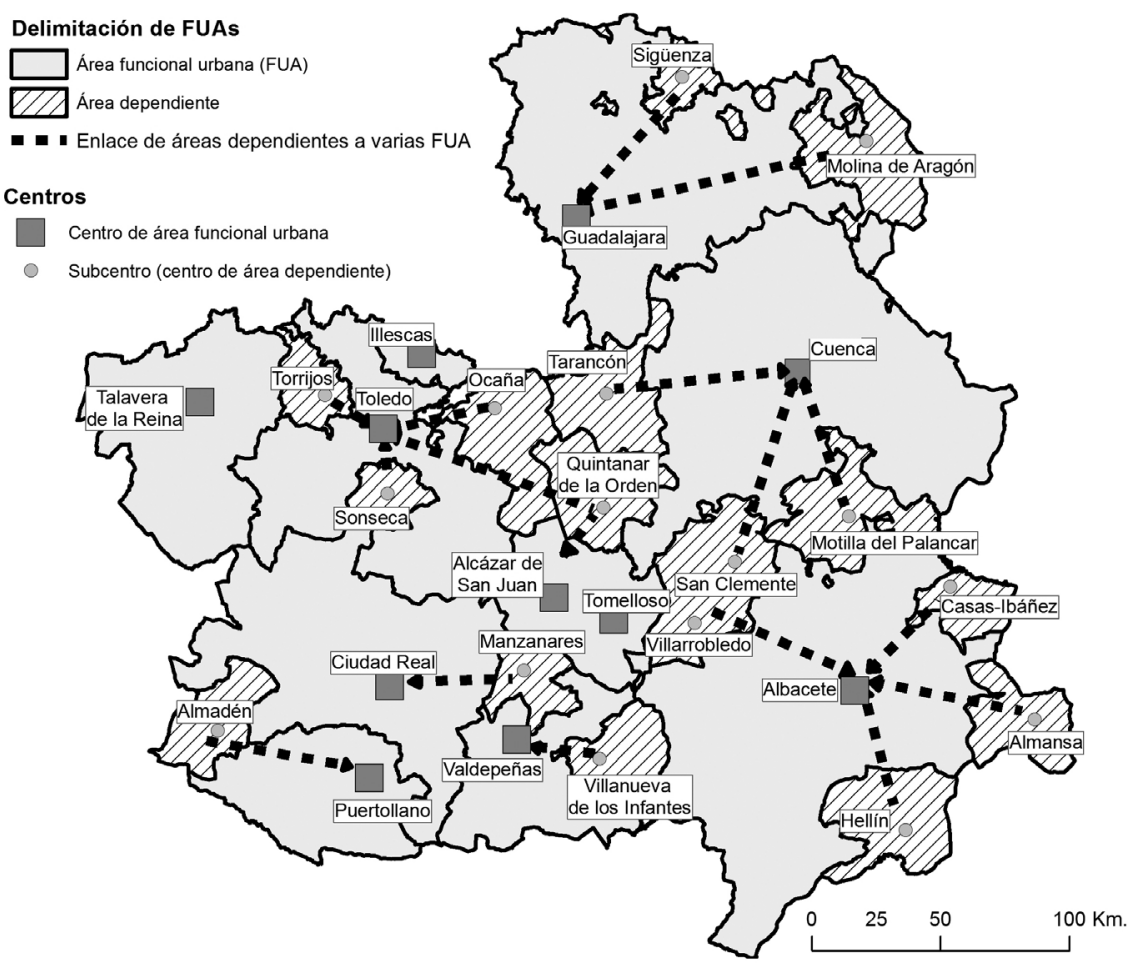

Figura 1. Áreas funcionales urbanas (FUA) en Castilla-La Mancha (España).

Fuente: Pillet et al., 2010. Adaptado. 
Para la definición de centralidades, como criterio, se establecen una batería de indicadores de servicios, públicos y privados, que permiten detectar los principales centros y subcentros y, al mismo tiempo, los distinguen en dos niveles en función de la especialización de los servicios. Posteriormente, emplean los flujos de población vinculada para establecer los límites de las FUA resultantes $^{2}$. Cabe señalar al respecto que, a pesar de utilizar criterios metodológicos muy diferentes, existe una cierta coincidencia, al destacar la importancia de los subcentros en la configuración del policentrismo utilizada por otros estudios, como es el caso del análisis del sistema urbano en España realizado por Roca, Moix y Arellano (2012).

A partir de aquí, se han establecido tres grandes conjuntos atendiendo a su naturaleza y a su ubicación: FUA localizadas en la periferia de Madrid, FUA de las capitales de provincia restantes y FUA vinculadas a ciudades medianas o pequeñas. En definitiva, unas muy dinámicas, en base a su crecimiento demográfico, la mayor parte de las cuales están en contacto con la Comunidad de Madrid, mientras que las que presentan crecimientos inferiores o negativos se encuentran en situación meridional y periférica, a las que se añaden las zonas industriales en crisis o en reestructuración, como la de Puertollano. En definitiva, un análisis que debe ser flexible, pues el AVE, las nuevas autovías, autopistas de peaje, etc. pueden producir cambios que deberán ser tenidos en cuenta posteriormente (Pillet et al., 2010). Apuntamos, no obstante, que aquellas FUA cercanas a la capital del Estado incluyen territorios que han manifestado un mayor dinamismo (Méndez y Prada, 2010) y albergan áreas que, progresivamente, se integran, también, en la región urbana policéntrica de Madrid (Solís, 2011).

\section{Las áreas funcionales urbanas (FUA) de Ciudad Real y Puertollano}

En este caso, dos de estas áreas funcionales urbanas o FUA serán objeto de análisis: la de Ciudad Real y la de Puertollano, ambas localizadas en la provincia de Ciudad Real. Antes de abordarlas, analizaremos las características generales de la red urbana castellano-manchega en la que ambas ciudades, también, presentan gran relevancia.

\subsection{Ciudad Real y Puertollano en la red urbana de Castilla-La Mancha}

Castilla-La Mancha no tiene un único núcleo rector capaz de organizar todo el territorio, por lo que se ha considerado una "región acéfala», y son las cinco capitales provinciales (Toledo, Ciudad Real, Albacete, Cuenca y Guadalajara), junto a otras ciudades importantes (Talavera de la Reina y Puertollano), las que estructuran la red urbana regional. Además, dada la cercanía de Madrid y su potencial de atracción, es clara su vinculación con el sistema urbano madrileño.

2. Una completa descripción del camino metodológico que lleva a esta delimitación se encuentra en Pillet et al. (2010). 
La red regional, con graves carencias infraestructurales y de comunicación, presenta una clara fragilidad estructural y falta de integración (Panadero y Pillet, 1999: 326; Cebrián, 2007a: 179-180; Pillet et al., 2010; Martínez, 2010), relacionadas con la localización espacial de los núcleos urbanos y la dispersión de los asentamientos, distribuidos de forma desigual por todo el territorio. Para su estructuración interna, necesita la complementación de sus ciudades con algunos núcleos más pequeños (semirurales y rurales), de modo que todo el espacio regional quede cubierto (Cañizares, 1999: 78).

En este contexto, son las provincias de Ciudad Real y de Toledo las que acumulan un mayor número de núcleos urbanos. En general, ciudades medias y pequeñas, como Ciudad Real y Puertollano, que, en los últimos años, al igual que otras en la región, «han acentuado su función de lugares centrales suministradores de servicios y constituyen los principales polos de atracción de la población castellano-manchega localizada en su entorno inmediato» (Panadero, García y Cañizares, 1999: 1172).

Analizamos, a continuación, las FUA conformadas por ambas ciudades, destacando que, en recientes estudios, se ha planteado su análisis conjunto para el estudio de la expansión del suelo urbano, principalmente discontinuo (Cebrián, García y Panadero, 2010: 25 y s.) o para destacar su integración territorial basada en la conexión a la alta velocidad ferroviaria, en proyectos comunes (algunos fallidos, como el aeropuerto Ciudad Real Central) y en la localización de nuevas empresas en el territorio intermedio, lo cual podría conducir a su fusión considerándolas como una única área funcional con dos centros en base a las relaciones de movilidad obligada entre sus ciudades principales (Coronado et al., 2011).

\subsubsection{La FUA de Ciudad Realy su área dependiente de primer nivel (Manzanares)} La FUA de Ciudad Real se estructura a partir de Ciudad Real como centro principal del área funcional y de Manzanares como centro de área dependiente de primer nivel.

Está organizada por la capital provincial, Ciudad Real, fundada en 1255, núcleo que evolucionó lenta y progresivamente como centro de servicios y se caracterizó más adelante por un importante deterioro urbanístico. Fue en el tránsito de las décadas de 1980 y 1990 cuando se benefició de la creación de un campus universitario (Universidad de Castilla-La Mancha) en 1985 y de la llegada del AVE en 1992, como motores de cambio, a los que se han unido otras mejoras en las vías de comunicación (Pillet, 1984; Pillet, 2012: 46-47). Actualmente, mantiene sus funciones terciarias (comercial, administrativa, educativo-cultural y sanitaria) (Rodríguez, 2012) y consolida su papel como capital provincial y ciudad más dinámica, de modo que es una de las capitales castellano-manchegas que mayor volumen de población vinculada alberga, junto con Toledo, la capital regional.

La FUA agrupa 43 municipios, con una población total de 200.379 habitantes en 2011 y un crecimiento de un 0,33\% para el período 2001-2011. El de su centro, Ciudad Real, con 74.798 habitantes en 2011, es algo superior (un 1,67\%) para el mismo período. Destaca la presencia de tres municipios 
que circundan el centro de la FUA (Ciudad Real) y recogen su expansión con crecimientos muy elevados, a pesar de no estar en la órbita de la Comunidad de Madrid: Miguelturra, con 14.517 habitantes y un 3,20\% de crecimiento real; Poblete, con 1.978 habitantes y un crecimiento real elevadísimo de un $8,12 \%$, y Cañada de Calatrava, con 115 habitantes y un $4,08 \%$ de crecimiento. A ellos unimos, en el extremo oriental, conectado con el centro a través de la autovía A-43, Daimiel, con 18.673 habitantes y un $0,88 \%$ de crecimiento real. Núcleos especialmente dinámicos que presentan un notable desarrollo de los servicios, como evidencian los datos correspondientes a los porcentajes de población afiliada a la Seguridad Social en este sector (tabla 1). Respecto al resto de municipios, cabe señalar que, en general, presentan crecimientos positivos, exceptuando algunos núcleos alejados de Ciudad Real e integrados en la zona de Los Montes, como Arroba de los Montes (un -1,83\%) y Alcoba (un -1,29\%), o bien con un importante peso de las actividades rurales, como es el caso de Granátula de Calatrava (un -1,20\%), entre otros.

El subcentro de la FUA, Manzanares, con 19.239 habitantes en 2011, organiza un área dependiente de primer nivel, agrupando en total 42.534 habitantes con sólo 4 municipios y un escaso crecimiento real en el período 2001-2011 (un 0,33\%).

Para finalizar, señalamos que si el centro de la FUA, Ciudad Real, agrupa el 37,3\% del total de población, el centro del área dependiente, Manzanares, supone algo más, un $45,2 \%$.

\subsubsection{La FUA de Puertollano y su área dependiente de segundo nivel (Almadén)} La FUA de Puertollano se estructura a partir de Puertollano como centro de área funcional y de Almadén, que genera un área dependiente de segundo nivel.

Está organizada por la ciudad de Puertollano, núcleo de origen medieval que experimentó un lento desarrollo hasta finales del siglo XIX, cuando se descubrió el carbón y se convirtió en una ciudad minera. Su crecimiento explosivo posterior se debió a la minería y a la llegada de actividades industriales a mediados del siglo XX (destilación de pizarras bituminosas, industrias petroquímicas y producción de energía), que la convirtieron en uno de los principales asentamientos de la provincia y de la región con una clara vocación industrial (Cañizares, 2001, 2010b). La crisis de la década de 1970 la relega a un segundo plano respecto a Ciudad Real y hoy subsiste dando un giro a sus actividades socioeconómicas en relación con las energías renovables (Cañizares, 2012: 99-100).

Puertollano, con una población total de 52.200 habitantes en 2011, es el centro de esta FUA. Es, también, la menor en número de municipios del conjunto regional, pues agrupa solo 11 y contabiliza 70.453 habitantes en 2011. Sobresale un hecho, el de registrar el crecimiento más bajo del conjunto de las FUA castellano-manchegas en el período 2001-2008 (Pillet et al., 2010), y alcanza también valores negativos para el período 2001-2011 (un -0,69\%), situación cercana a la que ofrece su propio centro, Puertollano, aunque con un crecimiento positivo de un $0,82 \%$. Con valores negativos, encontramos 
Tabla 1. Caracterización de los municipios integrados en la FUA de Ciudad Real

\begin{tabular}{|c|c|c|c|c|c|c|c|}
\hline FUA Ciudad Real & Pob. 2001 & Pob. 2011 & T.C.R. \% & Pob. Agr ${ }^{\star}$ & Pob. Ind* & Pob. Con* & Pob. Serv ${ }^{*}$ \\
\hline Abenójar & 1.681 & 1.605 & $-0,46$ & 34,97 & 9,09 & 18,18 & 37,76 \\
\hline Agudo & 1.987 & 1.866 & $-0,63$ & 36,36 & 9,55 & 11,14 & 42,95 \\
\hline Alcoba & 803 & 706 & $-1,29$ & 46,85 & 1,80 & 10,81 & 40,54 \\
\hline Alcolea de Ctrava & 1.608 & 1.616 & 0,05 & 18,18 & 12,34 & 11,69 & 57,79 \\
\hline Aldea del Rey & 2.109 & 1.969 & $-0,69$ & 36,36 & 8,15 & 20,06 & 35,42 \\
\hline Almagro & 8.537 & 9.083 & 0,62 & 5,12 & 20,59 & 7,61 & 66,68 \\
\hline Arenas de S. Juan & 1.050 & 1.077 & 0,25 & 38,67 & 3,11 & 23,11 & 35,11 \\
\hline Arroba de los M. & 608 & 506 & $-1,83$ & 60,38 & 5,66 & 5,66 & 28,30 \\
\hline Ballesteros de C. & 550 & 464 & $-1,70$ & 21,62 & 2,70 & 13,51 & 62,16 \\
\hline Bolaños de Cva. & 11.290 & 12.159 & 0,74 & 28,29 & 22,47 & 9,12 & 40,13 \\
\hline Cabezarados & 389 & 351 & $-1,03$ & 40,30 & 16,42 & 7,46 & 35,82 \\
\hline Calzada de Cva. & 4.574 & 4.430 & $-0,32$ & 21,57 & 9,93 & 9,80 & 58,69 \\
\hline Cañada de Ctrava & 76 & 115 & 4,08 & 44,44 & 0,0 & 0,0 & 55,56 \\
\hline Caracuel de Cva. & 172 & 168 & $-0,24$ & 52,00 & 0,0 & 0,0 & 48,00 \\
\hline Carrión de Ctrava & 2.644 & 3.003 & 1,27 & 11,46 & 4,28 & 27,35 & 56,91 \\
\hline Ciudad Real & 63.251 & 74.798 & 1,67 & 2,44 & 3,51 & 3,96 & 90,10 \\
\hline Corral de Ctrava & 1.278 & 1.172 & $-0,87$ & 19,56 & 21,78 & 16,44 & 42,22 \\
\hline Los Cortijos & 1.012 & 992 & $-0,20$ & 30,17 & 10,34 & 14,66 & 44,83 \\
\hline Daimiel & 17.095 & 18.673 & 0,88 & 8,55 & 25,80 & 14,10 & 51,55 \\
\hline Fernáncaballero & 1.073 & 1.143 & 0,63 & 10,32 & 2,85 & 10,68 & 76,16 \\
\hline Fontanarejo & 341 & 318 & $-0,70$ & 55,00 & 2,50 & 2,50 & 40,00 \\
\hline Fuente el Fresno & 3.476 & 3.650 & 0,49 & 32,66 & 7,89 & 21,98 & 37,46 \\
\hline Granátula de Cva. & 1.010 & 896 & $-1,20$ & 44,44 & 5,56 & 6,35 & 43,65 \\
\hline Horcajo de los M. & 1.060 & 1.019 & $-0,39$ & 21,93 & 5,88 & 17,11 & 55,08 \\
\hline Las Labores & 671 & 650 & $-0,32$ & 27,22 & 47,93 & 9,47 & 15,38 \\
\hline Luciana & 440 & 409 & $-0,73$ & 21,15 & 37,50 & 1,92 & 39,42 \\
\hline Malagón & 8.033 & 8.734 & 0,84 & 16,89 & 16,31 & 13,20 & 53,60 \\
\hline Miguelturra & 10.516 & 14.517 & 3,20 & 4,53 & 10,56 & 12,21 & 72,70 \\
\hline Navalpino & 292 & 267 & $-0,89$ & 28,57 & 0,0 & 21,43 & 50,00 \\
\hline Picón & 657 & 702 & 0,66 & 20,00 & 20,00 & 11,11 & 48,89 \\
\hline Piedrabuena & 4.671 & 4.815 & 0,30 & 13,03 & 14,33 & 15,85 & 56,79 \\
\hline Poblete & 836 & 1.978 & 8,12 & 7,63 & 14,87 & 6,46 & 71,04 \\
\hline Porzuna & 3.873 & 4.056 & 0,46 & 35,58 & 6,31 & 18,65 & 39,45 \\
\hline Pozuelo de Cva. & 2.571 & 3.204 & 2,19 & 14,60 & 27,49 & 17,87 & 40,03 \\
\hline Los Pozuelos & 522 & 444 & $-1,61$ & 49,00 & 3,00 & 12,00 & 36,00 \\
\hline Puebla de Don R. & 1.319 & 1.255 & $-0,50$ & 47,49 & 3,29 & 10,33 & 38,50 \\
\hline Retuerta del B. & 1.003 & 1.135 & 1,23 & 56,41 & 8,06 & 8,79 & 26,74 \\
\hline El Robledo & 1.102 & 1.307 & 1,70 & 32,08 & 9,17 & 22,50 & 36,25 \\
\hline Torralba de C. & 2.888 & 3.163 & 0,91 & 10,44 & 19,62 & 8,33 & 61,60 \\
\hline Valenzuela de C. & 830 & 770 & $-0,75$ & 25,47 & 6,60 & 15,09 & 53,83 \\
\hline Villar del Pozo & 111 & 98 & $-1,24$ & 3,03 & 0,0 & 27,27 & 69,70 \\
\hline Villarrubia de 0. & 9.722 & 11.096 & 1,32 & 22,51 & 12,24 & 25,28 & 39,97 \\
\hline \multicolumn{8}{|l|}{ Área Dep. de Manzanares } \\
\hline Llanos del C. & 726 & 708 & $-0,25$ & 84,87 & 3,29 & 5,26 & 6,58 \\
\hline Manzanares & 17.917 & 19.239 & 0,71 & 5,02 & 25,48 & 6,84 & 62,66 \\
\hline Membrilla & 6.591 & 6.349 & $-0,37$ & 34,68 & 12,93 & 7,71 & 44,67 \\
\hline La Solana & 15.047 & 16.238 & 0,76 & 26,00 & 13,29 & 14,82 & 45,90 \\
\hline Prov. CREAL & 478.581 & 530.175 & 1,02 & - & - & - & - \\
\hline $\mathrm{CLM}^{\star}$ & 1.755 .053 & 2.115 .034 & 1,86 & - & - & - & - \\
\hline España & 40.847 .371 & 47.190 .493 & 1,44 & _- & _- & - & _- \\
\hline
\end{tabular}

Fuente: INE y Servicio de Estadística de Castilla-La Mancha. CLM* Castilla-La Mancha.

Agr $^{*}$, Ind $^{*}$, Con $^{*}$, Serv $^{*}$ : porcentaje de afiliados a la Seguridad Social por sectores (agricultura, industria, construcción y servicios) en febrero de 2012. 
pequeños asentamientos de población aún muy ruralizados y alejados de Puertollano, como Hinojosas de Calatrava, con un $-2,75 \%$ de crecimiento real para el período 2001-2011, Fuencaliente, con un -1,51\%, y Cabezarrubias del Puerto, con un $-1,25 \%$. Por otra parte, aparecen valores positivos en aquellos núcleos más grandes, cercanos al centro, como Argamasilla de Calatrava, con $1,03 \%$, y Villamayor de Calatrava, con un $0,78 \%$, en los que, además, existe un volumen destacable de población empleada en el sector secundario y, en especial, en la industria (tabla 2).

Almadén, con solo 6.101 habitantes, actúa como subcentro y organiza un área dependiente de segundo nivel vinculada con dos FUA, la de Puertollano y también la de Ciudad Real (aunque se espera que la nueva autovía A-41 marque cada vez más su relación con Puertollano). Esta área dependiente agrupa 7 municipios en 2011, una población total de 10.939 habitantes con uno de los crecimientos negativos más relevantes de la región (un -1,33\%) y valores municipales significativos, como los de Alamillo, con un -2,21\%, Guadalmez, con un $-1,82 \%$, y Valdemanco de Esteras, con un $-1,73 \%$, en un territorio

Tabla 2. Caracterización de los municipios integrados en la FUA de Puertollano

\begin{tabular}{|c|c|c|c|c|c|c|c|}
\hline FUA Puertollano & Pob. 2001 & Pob. 2011 & TCR \% & Agr* & $\operatorname{lnd}^{*}$ & Con* & Serv* $^{*}$ \\
\hline Almodóvar del Campo & 7.139 & 6.711 & $-0,62$ & 15,59 & 15,01 & 23,71 & 45,69 \\
\hline Argamasilla de Calatrava & 5.361 & 5.941 & 1,03 & 3,58 & 31,05 & 25,46 & 39,91 \\
\hline Brazatortas & 1.176 & 1.117 & $-0,51$ & 29,12 & 8,79 & 15,93 & 46,15 \\
\hline Cabezarrubias & 621 & 548 & $-1,25$ & 27,45 & 3,92 & 43,14 & 25,49 \\
\hline Fuencaliente & 1.293 & 1.112 & $-1,51$ & 38,86 & 5,71 & 7,43 & 48,00 \\
\hline Hinojosas de Calatrava & 711 & 539 & $-2,75$ & 23,73 & 10,17 & 11,86 & 54,24 \\
\hline Mestanza & 865 & 815 & $-0,60$ & 43,40 & 2,83 & 15,09 & 38,68 \\
\hline Puertollano & 48.086 & 52.200 & 0,82 & 1,00 & 31,92 & 9,34 & 57,74 \\
\hline Solana del Pino & 502 & 427 & $-1,61$ & 33,33 & 3,17 & 9,52 & 53,97 \\
\hline Villamayor de Calatrava & 615 & 665 & 0,78 & 25,61 & 25,61 & 3,66 & 45,12 \\
\hline Villanueva de San Carlos & 435 & 378 & $-1,40$ & 39,06 & 4,69 & 1,56 & 54,69 \\
\hline \multicolumn{8}{|l|}{ Área Dep. de Almadén } \\
\hline Alamillo & 663 & 531 & $-2,21$ & 34,74 & 0,94 & 4,48 & 33,18 \\
\hline Almadén & 6.830 & 6.101 & $-1,13$ & 8,41 & 13,50 & 6,95 & 71,14 \\
\hline Almadenejos & 518 & 502 & $-0,31$ & 50,59 & 0,0 & 20,00 & 29,41 \\
\hline Chillón & 2.275 & 2.052 & $-1,03$ & 14,05 & 5,48 & 22,62 & 57,86 \\
\hline Guadalmez & 1.049 & 874 & $-1,82$ & 34,31 & 8,76 & 10,95 & 45,99 \\
\hline Saceruela & 713 & 642 & $-1,05$ & 53,33 & 0,95 & 8,57 & 37,14 \\
\hline Valdemanco del Esteras & 282 & 237 & $-1,73$ & 65,38 & 0,0 & 0,0 & 34,62 \\
\hline Prov. C. Real & 478.581 & 530.175 & 1,02 & - & - & - & - \\
\hline $\mathrm{CLM}^{*}$ & 1.755 .053 & 211.534 & 1,86 & - & - & - & - \\
\hline España & 40.847 .371 & 47.190 .493 & 1,44 & - & - & - & - \\
\hline
\end{tabular}

Fuente: INE y Servicio de Estadística de Castilla-La Mancha. CLM* Castilla-La Mancha.

Agr ${ }^{\star}$, Ind $^{\star}$, Con$^{*}, S_{\text {Serv*}}^{*}$ porcentaje de afiliados a la Seguridad Social por sectores (agricultura, industria, construcción y servicios) en febrero de 2012. 
tradicionalmente vinculado a la minería (hoy clausurada) y a las actividades agrarias.

Por último, si en la FUA de Puertollano el peso de su centro es casi absoluto (un $73,9 \%$ ) sobre el total de población, en el área de Almadén, el subcentro ${ }^{3}$ representa solo el $55,7 \%$.

\subsection{El corredor Ciudad Real-Puertollano, un espacio de conexión entre ambas FUA}

En Castilla-La Mancha, no se ha abordado plenamente la ordenación territorial desde el marco institucional, donde «la planificación urbanística y sectorial ha sido primordial en los desarrollos legislativos, no siendo hasta fechas recientes cuando se observa un cierto giro espacial» (Plaza, Martínez y Gosálvez, 2010: 519). Es a finales de la pasada década cuando la Consejería de Ordenación del Territorio y Vivienda intenta responder a la aplicación de la Estrategia Territorial Europea (1999), del Convenio Europeo del Paisaje (en vigor para España desde 2008) y el Libro Verde sobre la Cohesión Territorial (CCE, 2008), con la elaboración del Plan de Ordenación del Territorio «Estrategia Territorial de Castilla-La Mancha», aprobado inicialmente en 2009, cuya finalidad es establecer un modelo territorial definitorio de la organización racional y equilibrada del suelo y de los recursos naturales, procurando la articulación, la integración y la cohesión de la comunidad autónoma, tanto interna como con el resto de España, en especial con las comunidades limítrofes ${ }^{4}$. De él se derivaban cinco planes de ordenación del territorio o POT subregionales, centrados en sectores concretos de la región (actualmente paralizados después del cambio de gobierno en 2011), entre los que se encontraba el POT «Corredor Ciudad Real-Puertollano" (también aprobado inicialmente en 2009).

El mismo nombre de corredor ya indica que el criterio que prevaleció en su caracterización era un intento de armonizar los procesos que se estaban desarrollando en una de las áreas urbanas dinámicas de la región de Castilla-La Mancha, donde, en los últimos años, a partir del peso específico de la capital provincial, Ciudad Real, se ha venido construyendo una nueva realidad territorial a modo de espacio de acumulación: el eje formado por Ciudad Real y Puertollano (Consejería de Ordenación del Territorio y Vivienda, 2009b). Se trata de un eje especialmente dinámico que actúa como conector de las dos FUA analizadas, independientemente de la planificación. Concentra 176.289 habitantes en 2011 (un 33,25\% de la provincia de Ciudad Real y un 8,33\% del total de población de Castilla-La Mancha) y abarca $1.719,15 \mathrm{~km}^{2}$ (un 8,7\% de

3. Cabe señalar que, en Castilla-La Mancha, los subcentros con área dependiente de segundo nivel organizan pequeñas áreas de influencia que merecen ser destacadas para la vertebración del territorio y, sobre todo, para buscar un desarrollo territorial equilibrado y que llegue, como manifiesta la ETE, hasta todos los rincones de la comunidad autónoma.

4. Su ámbito de referencia es la Ley $2 / 1998$, de 4 de junio, de ordenación del territorio y de la actividad urbanística (LOTAU) de Castilla-La Mancha y su último texto refundido (TRLOTAU) de 2010. 


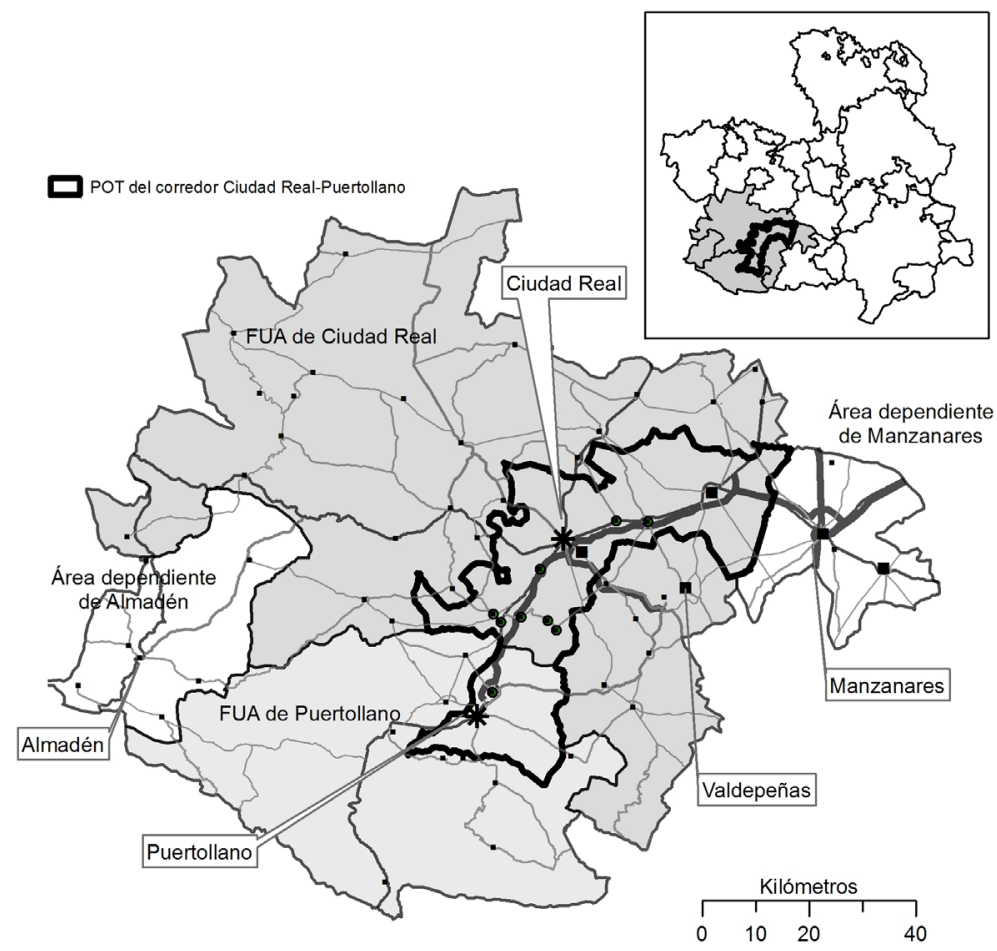

Figura 2. Localización del corredor Ciudad Real-Puertollano en el contexto de ambas áreas funcionales urbanas (FUA) en Castilla-La Mancha.

Fuente: Pillet et al., 2010. Elaboración propia.

la provincia de Ciudad Real y un 2,1\% de la región), con una densidad demográfica de 102,5 habitantes $/ \mathrm{km}^{2}$ (figura 2) ${ }^{5}$. Desde el punto de vista territorial, destaca la preeminencia de sus núcleos urbanos más grandes, como Ciudad Real (al que se vincula Miguelturra), Puertollano (al que se vincula Argamasilla de Calatrava) y Daimiel, con una base económica más diversificada, frente a los más pequeños en los que todavía se manifiesta la importancia de las actividades rurales en el mercado de trabajo (Cañizares, 2010a: 161-162).

La planificación regional lo incluye dentro de las áreas de acumulación urbana y, más concretamente, de los centros regionales y sus áreas de influencia, zonas relacionadas con fenómenos metropolitanos originados tanto de forma radial con respecto a una ciudad (como son también los casos de Tala-

5. Agrupa los términos municipales de Argamasilla de Calatrava, Ballesteros de Calatrava, Cañada de Calatrava, Caracuel de Calatrava, Carrión de Calatrava, Ciudad Real, Corral de Calatrava, Daimiel, Miguelturra, Poblete, Puertollano, Torralba de Calatrava y Villar del Pozo. 
vera y de Cuenca), como en torno a ejes entre polos de actividad (el corredor Puertollano-Ciudad Real-Daimiel y el corredor en forma de Y que forman Albacete-Hellín-Almansa). En estas zonas, las ciudades poseen sus propias especificidades, aunque destaca su especialización en dar cobertura de servicios públicos y privados a sus zonas de influencia, combinada con actividades industriales, construcción y, en menor medida, cierta producción agropecuaria (Consejería de Ordenación del Territorio y Vivienda, 2009a: 114).

\section{La conexión entre Ciudad Real y Puertollano y sus comunicaciones}

Una de las singularidades del espacio ocupado por las FUA es la progresiva acumulación de la oferta de transporte que ha ido consolidando una estructura territorial de tipo corredor, en la medida que las ciudades de Ciudad Real y Puertollano han ido fortaleciendo sus relaciones y extendiéndolas progresivamente, aunque en el pasado no han colaborado ni se han complementado especialmente.

Las infraestructuras de transporte, que desarrollaremos a continuación, son las que le dan cohesión (Cañizares, 2009), sobre todo en la zona interior correspondiente al corredor. Nos referimos, principalmente, a la línea de alta velocidad ferroviaria (AVE) Madrid-Sevilla, con paradas en Ciudad Real y Puertollano; las autovías A-41 y A-43 en la conexión Valencia-Lisboa; la futura AP-41, que unirá Toledo, Ciudad Real y Córdoba, y la reciente autovía CM-45 Ciudad Real-Valdepeñas (Autovía del IV Centenario); añadimos a ellas el aeropuerto Central Ciudad Real, inaugurado en 2008 como proyecto de singular interés regional, pero clausurado en octubre de 2011 ante la falta de negocio.

\subsection{La estructura de transporte y su evolución}

El proceso de consolidación de la conexión entre las ciudades de Ciudad Real y Puertollano ha experimentado una secuencia en la que dos hechos han sido especialmente protagonistas: la llegada de la alta velocidad ferroviaria, en 1992, y la consolidación paulatina de un eje de gran capacidad viaria. El primer factor ubica a Ciudad Real y Puertollano en el mapa de las comunicaciones nacionales, mientras que el segundo es un proceso que comienza a modificar la estructura de las relaciones intraprovinciales y regionales, primero, con la apertura del tramo de la A-41 entre Ciudad Real y Puertollano, en 2006, y, posteriormente, con diversas prolongaciones (A-43) hasta la actual consolidación.

La estructura de las comunicaciones en Castilla-La Mancha viene determinada por su papel secundario dentro del sistema español y por el hecho de que la primera jerarquía de la red no ha sido pensada para integrar los espacios regionales (Martínez, 2010, 2012). En la provincia de Ciudad Real, la decisión de aprovechar el paso de Despeñaperros para las comunicaciones entre el centro peninsular y Andalucía supuso que el centro de gravedad provincial se apartara unos 40 kilómetros de su capital (Menéndez, 2004), tanto en lo 
referido al corredor preferente de carreteras (la anterior N-IV, hoy A-4), como de ferrocarriles (línea Madrid-Andalucía).

La construcción de la línea de alta velocidad ${ }^{6}$ (LAV) Madrid-Sevilla y la decisión de crear el nuevo acceso ferroviario a Andalucía (NAFA) favoreció a Ciudad Real y Puertollano, que se integraron en el nuevo mapa ferroviario español de la mano de un nuevo modo de transporte (Menéndez et al., 2002), con lo cual se inició una nueva etapa caracterizada por un cambio de inercia, dadas las nuevas oportunidades que se vislumbraban, esencialmente en las comunicaciones con Madrid, tanto para Ciudad Real como para Puertollano.

Con esta nueva oferta, se inició rápidamente una etapa de oportunidades en ambas ciudades, al situarse en una escala de conexión con Madrid inferior a una hora de viaje, lo que modificaba el potencial de relaciones a escala nacional de forma neta. No obstante, Ciudad Real ha desarrollado procesos de cambio en los que la cercanía con Madrid ha desempeñado un papel importante (Pillet, 2005; Garmendia, 2007; Garmendia et al., 2011), mientras que el grado de aprovechamiento en Puertollano ha sido algo menor, debido a la situación socioeconómica local (Menéndez et al., 2002).

Cronológicamente, la alta velocidad ferroviaria (AVF) supone el comienzo de un cambio de inercia territorial, pero no lo explica por completo. A ello debemos unir la mejora en las conexiones de orden regional y local que vienen de la mano de las transformaciones dentro de la red de carreteras. El Gobierno español plantea, en 1993, su Plan Director de Infraestructuras 1993-2007, que tiene por objetivo primordial, en materia de carreteras, completar la estructura radial de las comunicaciones peninsulares y formar una malla a través de ejes complementarios. Las sucesivas planificaciones que se van sustituyendo mantienen este objetivo e introducen la necesidad de asentar una red de vías de gran capacidad para permitir la máxima interconectividad posible, siguiendo la jerarquía urbana provincial. A los tradicionales ejes centro-periferia, establecidos desde la época de Carlos III, se unen nuevos ejes trasversales, además de radiales, que duplican en parte la oferta existente y se conciben como medio para una mejor difusión de los flujos que atraviesen el centro peninsular. Ambos objetivos confluyen en la zona de estudio, puesto que tanto el eje Madrid-Córdoba (descongestión de la A-4 a través de una autopista de peaje: AP-41), como el eje Lisboa-Valencia (red complementaria: A-43) confluyen en el centro de la provincia de Ciudad Real. Un rápido compromiso adquirido permite construir un tramo libre de peaje de la futura AP-41 por el desdoblamiento de la $\mathrm{N}-420$ (por eso es libre de peaje y recibe el nombre de A-41) entre Ciudad Real y Puertollano. Sus 36,9 km aumentan la integración en la zona y comienzan a consolidar una estructura de tipo corredor que ya se había empezado a apuntar con la llegada del AVE. La apertura posterior de varios tramos de la A-43, primero, desde Ciudad Real hasta Daimiel, luego, Manzanares (conexión con la A-4) y, posteriormente, Tomelloso (CM-42) y Atalaya del Cañavate (AP-36, A-3 y A-31) han incrementado de forma eviden-

6. La alta velocidad ferroviaria se identifica con las siglas AVF o AV. 
te la capacidad conectiva y la inserción de ambas ciudades y el eje resultante. El esquema final de conexiones se resume en la figura 3.

Por tanto, nos encontramos con dos ciudades que, antes de la consolidación de estas nuevas infraestructuras de transporte, se comportaban, respectivamente, como importantes centros, con sus áreas de influencia y dinámicas sociales y económicas respectivas, pero relativamente desconectados de otras áreas provinciales, regionales y nacionales; y hoy se consideran "conectadas" dentro de la jerarquía urbana regional, es decir, aquellas bien posicionadas en la red nacional de transportes por carretera y ferrocarril, que cuentan, además, con iniciativas para reforzar su capacidad de relación (Cebrián, 2007b: 26).

La rápida inserción y el éxito de los servicios de AVF entre Ciudad Real y Madrid y, en menor medida, entre Puertollano y Madrid, además del sorprendente dinamismo de la conexión entre Puertollano y Ciudad Real (15 minutos de trayecto) comenzó a generar un flujo de relaciones que la autovía ha reforzado. Las oportunidades acumuladas a lo largo de este eje de gran capacidad han suscitado expectativas en materia de suelo industrial (Santos y Martínez,

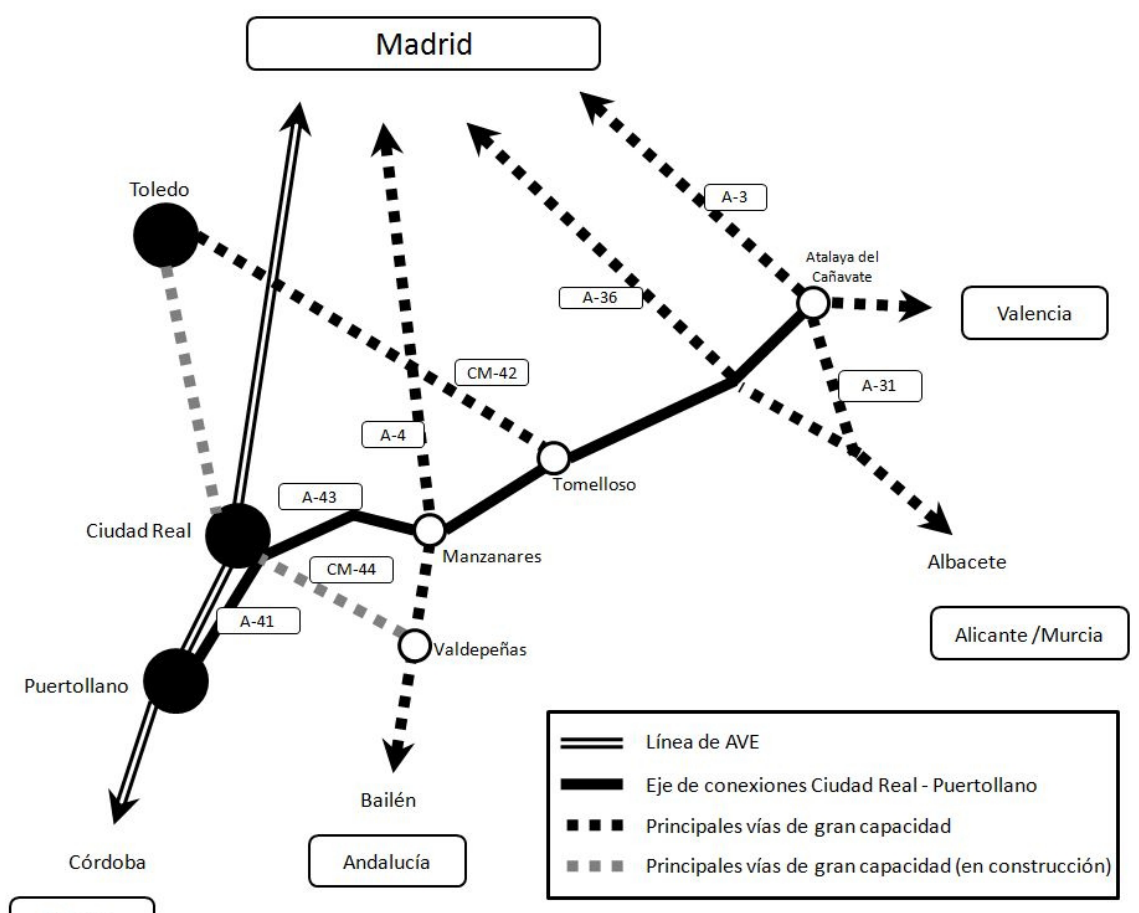

Sevilla

Figura 3. Esquema de conexiones del eje Ciudad Real-Puertollano (Castilla-La Mancha). Fuente: elaboración propia. 
2010) y es la base sobre la que se fundamentó posteriormente la elaboración del ya analizado Plan de Ordenación del Territorio «Corredor Ciudad RealPuertollano", que se prolongó por la A-43 hasta Daimiel. Además, bajo el argumento de las comunicaciones privilegiadas, se justificaba la oportunidad del fracasado proyecto del aeropuerto Ciudad Real-Central, que señalaba como principal activo la capacidad conectiva disponible, con acceso directo a AVE, ferrocarril convencional y dos ejes de carreteras de gran capacidad (la ya existente A-41 y la CM-44 en proceso de finalización).

\subsection{Las relaciones entre Ciudad Real y Puertollano}

La relación funcional entre ambas ciudades no ha sido especialmente intensa históricamente, como ya hemos señalado, y se ha circunscrito prácticamente a cuestiones de carácter administrativo derivadas de la capitalidad provincial de Ciudad Real. Cada una de ellas ha desarrollado sus áreas funcionales respectivas, basadas en la capacidad de generar centralidad en materia de servicios, centros comerciales y como vertebradoras de sus mercados laborales correspondientes (Pillet et al., 2010). Analizaremos, a continuación, las relaciones que se establecen entre ambas en dos aspectos de especial relevancia: los flujos de población vinculada y la conexión de alta velocidad ferroviaria.

\subsubsection{Los flujos de población vinculada}

El estudio de la población vinculada ha sido señalada como una importante fuente de información para el análisis territorial (Vinuesa, 2005), y se ha aplicado en varias ocasiones para caracterizar las relaciones entre núcleos a través de los flujos de personas que se desplazan en el espacio (Pons et al., 2009); en Castilla-La Mancha ha sido utilizada para definir los centros (Pillet et al., 2007) y delimitar las áreas funcionales urbanas (Pillet et al., 2010). Esta información se desagrega en tres componentes que definen, asimismo, tres tipos de vinculación, por razones de trabajo (laborales), estudios o residencia. En los tres casos, encontramos contabilizadas personas que residen en un municipio y declaran vincularse a otro por estos tres motivos, pero se desconocen más detalles sobre las características de dichas vinculaciones, frecuencias, motivos, etc.

Los datos del censo de 2001 permiten comprobar la importancia de Ciudad Real y Puertollano, por la entidad y variedad de los flujos establecidos. Observando las primeras quince relaciones de vinculación que reciben ambas ciudades (tablas 3 y 4 ), podemos realizar una primera reflexión sobre el diferente potencial de relación de cada una de ellas y su estructura. Ciudad Real supera en más de cuatro veces a Puertollano en población vinculada, ello refuerza dos hechos: su condición de capital provincial y su localización más centrada en el territorio, y supera, por ejemplo, a Albacete, que es la ciudad más poblada de la región (Pillet et al., 2007). También sobresale la proporcionalidad de estos flujos. Ciudad Real destaca por relaciones de trabajo y estudios, que se encuentran en el mismo orden de magnitud, mientras que Puertollano mantiene relaciones de segunda residencia y trabajo, preferentemente. 
En Ciudad Real (figura 4), identificamos tres grupos de relaciones según su entidad y su composición: las relaciones preferentes, dominadas por los tres primeros núcleos, Miguelturra, Puertollano y Madrid, entre los que destacamos la intensidad del flujo con Miguelturra, municipio separado apenas $3 \mathrm{~km}$ y con

Tabla 3. Principales núcleos emisores de población vinculada hacia Ciudad Real (2001)

\begin{tabular}{lrrrr}
\hline & Trabajo & Residencia & Estudios & \multicolumn{1}{c}{ Total } \\
\hline Ciudad Real & 8.548 & 6.161 & 8.418 & 23.127 \\
Miguelturra & 2.002 & 62 & 358 & 2.422 \\
Puertollano & 495 & 205 & 1.151 & 1.851 \\
Madrid & 261 & 1.437 & 38 & 1.736 \\
Daimiel & 436 & 61 & 351 & 848 \\
Carrión de Calatrava & 358 & 51 & 167 & 576 \\
Malagón & 273 & 21 & 185 & 479 \\
Valdepeñas & 102 & 29 & 328 & 459 \\
Manzanares & 126 & 23 & 309 & 458 \\
Bolaños de Calatrava & 138 & 89 & 209 & 436 \\
Calzada de Calatrava & 242 & 71 & 104 & 417 \\
Torralba de Calatrava & 260 & 57 & 85 & 402 \\
Almagro & 193 & 31 & 172 & 396 \\
Piedrabuena & 189 & 26 & 169 & 384 \\
Alcázar de San Juan & 71 & 22 & 229 & 322 \\
Pozuelo de Calatrava & 188 & 22 & 108 & 318 \\
\hline
\end{tabular}

Fuente: Censo 2001. Elaboración propia.

Tabla 4. Principales núcleos emisores de población vinculada hacia Puertollano (2001)

\begin{tabular}{lrrrr}
\hline & Trabajo & Residencia & Estudios & Total \\
\hline Puertollano & 2.491 & 2.469 & 441 & 5.401 \\
Argamasilla de Calatrava & 531 & 61 & 165 & 757 \\
Almodóvar del Campo & 561 & 36 & 101 & 698 \\
Madrid & 104 & 512 & 0 & 616 \\
Ciudad Real & 422 & 109 & 29 & 560 \\
Hinojosas de Calatrava & 43 & 56 & 15 & 114 \\
Cabezarrubias del Puerto & 34 & 60 & 12 & 106 \\
Brazatortas & 47 & 24 & 30 & 101 \\
Miguelturra & 14 & 66 & 2 & 82 \\
Leganés & 5 & 66 & 0 & 71 \\
Calzada de Calatrava & 58 & 7 & 5 & 70 \\
Valdepeñas & 12 & 34 & 1 & 47 \\
Daimiel & 38 & 0 & 3 & 41 \\
Mestanza & 11 & 5 & 22 & 38 \\
Getafe & 5 & 32 & 0 & 37 \\
Alcalá de Henares & 4 & 31 & 0 & 35 \\
\hline
\end{tabular}

Fuente: Censo 2001. Elaboración propia. 


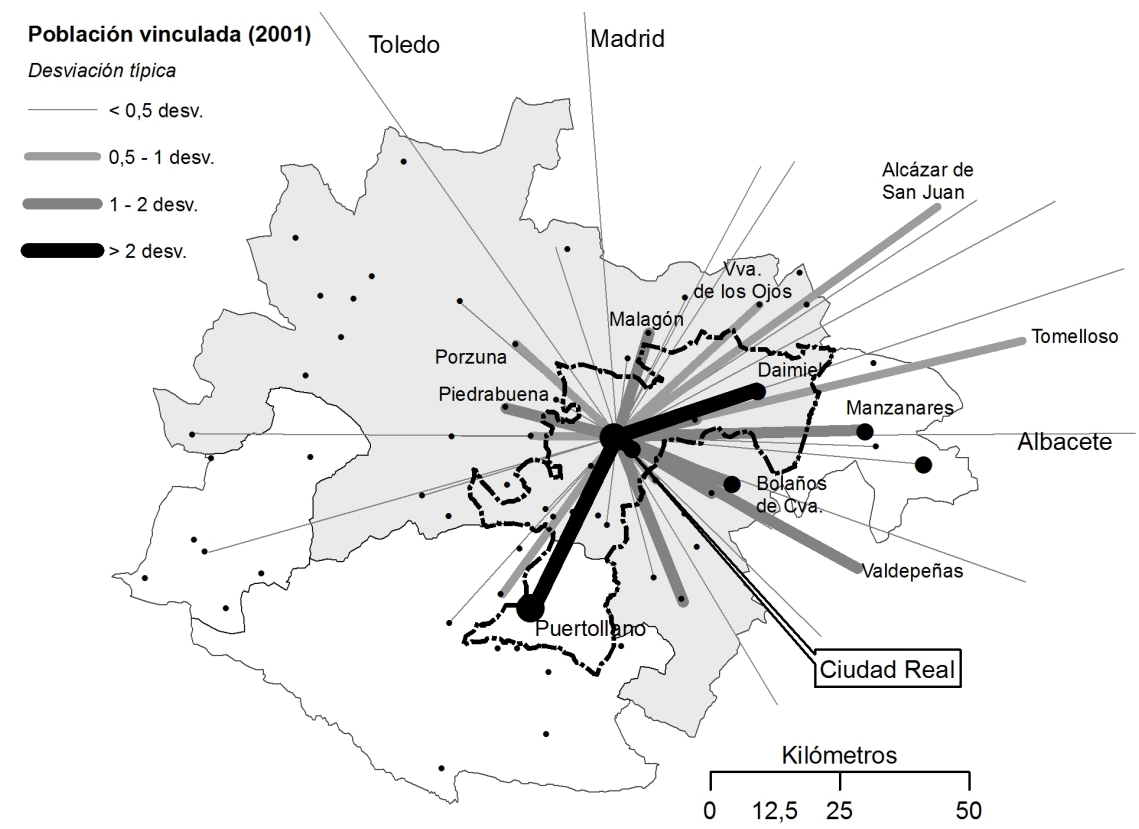

Figura 4. Flujos de población vinculada con destino en Ciudad Real.

Fuente: Censo 2001. Elaboración propia.

el que existe un evidente proceso de integración urbana (Rodríguez, 2012); en la relación con Puertollano es muy importante el apartado de estudios, mientras que con Madrid sobresale la segunda residencia, en la que el AVE ha tenido mucha influencia (Garmendia, 2007). En segundo lugar, aparecen relaciones de proximidad basadas en temas laborales con municipios que tienen a Ciudad Real como centro económico esencial que articula el mercado laboral de la zona, todos ellos dentro de su FUA y, ocasionalmente, en el corredor. Finalmente, encontramos relaciones de media distancia con centros y subcentros adyacentes entre los que aparecen otros núcleos externos, centros o subcentros de otras FUA, que mantienen importantes flujos por estudios con Ciudad Real, muestra de la presencia del campus universitario y su importancia en el contexto provincial y regional.

Puertollano tiene otra lógica interpretativa (figura 5), ya que la diferencia de magnitud en sus cifras indica una intensidad de relaciones y una distribución jerárquica más atenuada. Destacan las relaciones por trabajo, con el factor distancia como principal variable condicionada, y la segunda residencia, en la que el arco territorial se abre algo más, en este caso aparecen tanto Madrid como algunos municipios de su área metropolitana (Getafe, Leganés, Alcalá de Henares). Además, cuatro municipios dominan las relaciones con Puertollano: 


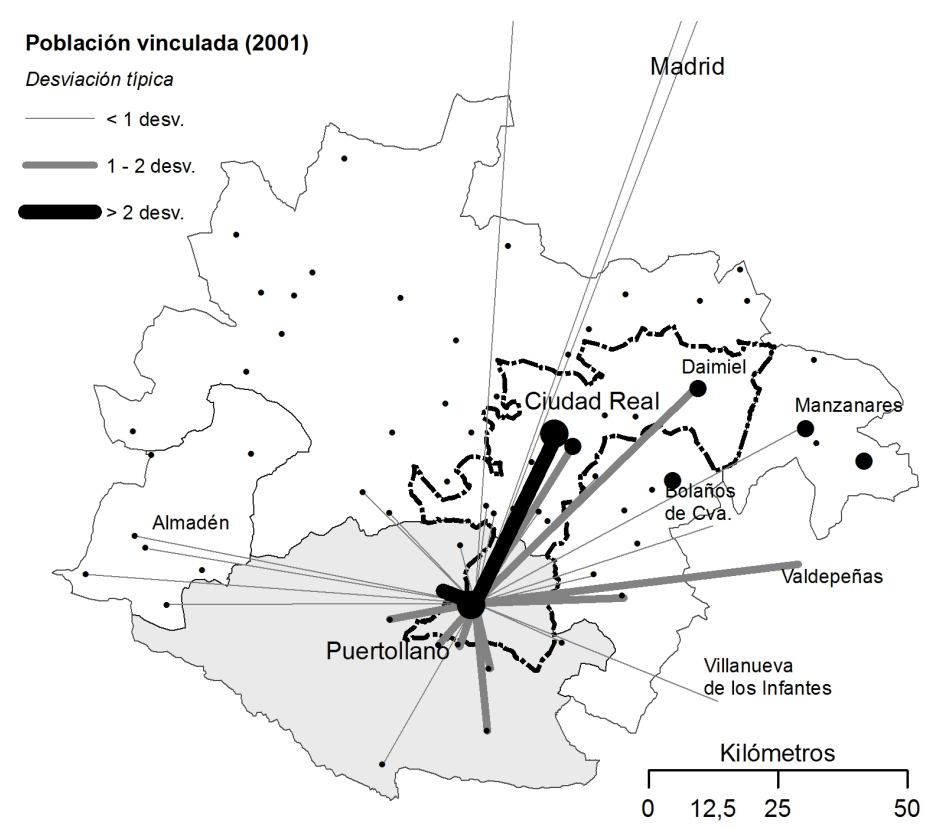

Figura 5. Flujos de población vinculada con destino en Puertollano.

Fuente: Censo 2001. Elaboración propia.

Almodóvar del Campo, Argamasilla de Calatrava, Ciudad Real y Madrid, a partir de los cuales existe un importante escalón en la potencia de los flujos. Puertollano, por tanto, combina la jerarquía local y comarcal (Almodóvar del Campo y Argamasilla de Calatrava) con las relaciones funcionales de escala regional (Ciudad Real y Madrid).

En síntesis, se aprecia un flujo intenso de relaciones en el corredor, aunque no se circunscriben a éste en exclusiva. Evidentemente, el tamaño de los núcleos influye en su capacidad de interrelación, y las zonas periféricas y la mitad oeste de las FUA están dominadas por pequeños municipios que no establecen grandes flujos de población vinculada. Esto polariza las relaciones en dirección al eje del corredor y las ciudades pequeñas de Valdepeñas, Manzanares o Bolaños. Por último, cabe destacar, una vez más, la presencia de Madrid entre las relaciones de mayor jerarquía en ambos casos, lo cual demuestra la influencia que las conexiones de AVE tienen en la configuración de las relaciones del corredor y sus centros.

\subsubsection{El ferrocarril de alta velocidad y su papel en el corredor}

Como se ha señalado en el apartado anterior, la llegada del ferrocarril de alta velocidad en 1992 comienza a modificar esta inercia. La interrelación entre 
ambas ciudades se ha ido intensificando con esta nueva oferta, y, ya en 1995, los viajeros anuales del servicio Puertollano-Ciudad Real-Madrid superaron el millón (Menéndez et al., 2002). El tipo de operación protagonista en este periodo fue el llamado AVE Lanzadera, un servicio de media distancia de altas prestaciones que se prolongó hasta 2005 y que privilegió este corredor desde el punto de vista de la política tarifaria (altamente subvencionado) y de frecuencias (hasta 20 operaciones diarias en ambos sentidos). Desde 2005 hasta 2009, se sucedieron varias decisiones que modificaron las condiciones iniciales del servicio, y se creó un modelo de negocio más rígido y menos subvencionado.

La tabla 5 refleja la evolución reciente de viajeros en esta conexión, lo cual ofrece diversas consideraciones de interés: en primer lugar, la proporcionalidad entre ambas es del orden del $72 \%$ al $74 \%$ a favor de Ciudad Real, sabiendo que, en la dinámica evolutiva, destaca la tendencia general descendente en la demanda para los cuatro años considerados, reflejo de la disminución progresiva de la demanda relacionada con la coyuntura económica regresiva. En ese sentido, conviene apuntar que el peso de Ciudad Real en la proporción ha aumentado dos puntos en estos cuatro años. El servicio dominante es, sin lugar a dudas, la AV Media Distancia (Avant), con prácticamente el 90\% de la demanda en la línea. Es más destacado el peso en el caso de Puertollano que en el de Ciudad Real, que diversifica algo más su demanda.

Sobresale, también, el incremento de los servicios convencionales (ancho español y velocidad normal) de media distancia, que experimentan crecimientos impresionantes en los datos de 2010 respecto a 2009, de modo que se superan con creces incluso los valores de 2007. Cabe interpretar que esta demanda se asume derivada de los servicios de AV hacia servicios convencionales, menos competitivos en tiempo y frecuencias, pero más baratos.

Tabla 5. Viajeros de ferrocarril en la línea Madrid-Puertollano-Ciudad Real (2007-2010)

\begin{tabular}{|c|c|c|c|c|c|c|c|c|}
\hline & \multicolumn{4}{|c|}{ Viajeros } & \multicolumn{4}{|c|}{ Porcentajes } \\
\hline & 2007 & 2008 & 2009 & 2010 & 2007 & 2008 & 2009 & 2010 \\
\hline Madrid-Ciudad Real & 1.028 .064 & 976.912 & 1.005 .579 & 922.915 & 72 & 73 & 74 & 74 \\
\hline AV Larga Distancia & 65.178 & 70.854 & 61.681 & 47.491 & 5 & 5 & 5 & 4 \\
\hline AV Media Distancia & 923.452 & 904.748 & 943.607 & 817.427 & 64 & 68 & 70 & 65 \\
\hline Larga Distancia & 24.418 & 326 & & & 2 & 0,02 & 0 & 0 \\
\hline Media Distancia & 15.016 & 984 & 291 & 57.997 & 1 & 0,07 & 0,02 & 5 \\
\hline Madrid-Puertollano & 409.232 & 357.941 & 349.631 & 328.457 & 28 & 27 & 26 & 26 \\
\hline AV Larga Distancia & 23.189 & 24.255 & 18.840 & 13.993 & 2 & 2 & 1 & 1 \\
\hline AV Media Distancia & 370.531 & 333.191 & 330.672 & 295.536 & 26 & 25 & 24 & 24 \\
\hline Larga Distancia & 9.032 & 134 & & & 1 & 0,01 & 0 & 0 \\
\hline Media Distancia & 6.480 & 361 & 119 & 18.928 & 0 & 0,03 & 0,01 & 2 \\
\hline
\end{tabular}

Total (Madrid-Ciudad

Real-Puertollano)

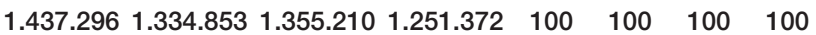

Fuente: Fundación de los Ferrocarriles Españoles, 2011: 80-81. Elaboración propia. AV: Alta Velocidad. 
El peso de esta conexión en las dinámicas locales de Ciudad Real y Puertollano es indiscutible, si comparamos el volumen de viajeros con la población residente en ambas ciudades, y más destacado aún en el caso de Ciudad Real. En las dos décadas que estos servicios han estado operando, se ha generado una importante relación funcional, en primer lugar con Madrid, ya que ambas ciudades se encuentran ahora en tiempos de ida y vuelta asimilables a los trabajadores pendulares de las áreas metropolitanas tradicionales (55 y 70 minutos a Ciudad Real y Puertollano, respectivamente). Y, en segundo lugar, las relaciones entre ellas también se han visto reforzadas. En la primera fase de AVE Lanzadera (de 1992 a 2005), las condiciones para los viajeros entre las dos ciudades era realmente ventajosa, con la opción de bonos mensuales, de 50 o de 20 viajes sin necesidad de reserva previa y con descuentos tarifarios importantes. El Avant, en 2005, que sustituyó al AVE Lanzadera, supuso un incremento en la rigidez del modelo, con la necesidad de realizar reserva previa y el aumento progresivo de tarifas, y coincidió, además, con la apertura al tráfico de la A-41. Parte de la demanda previa de los servicios Lanzadera se pudo ver reconducida por la nueva vía de gran capacidad, además de una demanda generada por las nuevas oportunidades.

\section{Conclusiones}

Los objetivos planteados por la Agenda Territorial Europea 2020 (UE, 2011) sólo podrán alcanzarse si se tiene en cuenta la dimensión territorial, puesto que las oportunidades de desarrollo son diferentes para cada región dentro de la Unión Europea en función de sus potencialidades, y es la cohesión territorial, ahora unida a la cohesión económica, objetivo común para todas ellas.

En este contexto, centrándonos en la región de Castilla-La Mancha, en el interior de la península Ibérica, y concretamente en la provincia de Ciudad Real, consideramos importante establecer la complementariedad entre las áreas funcionales urbanas o FUA de Ciudad Real y de Puertollano. Dos ciudades, funcionalmente diferentes, que organizan actualmente dos de las diez áreas funcionales delimitadas para esta región, potencialmente complementarias y favorecidas por las vías de comunicación y los flujos de población vinculada (analizados según los datos del Censo Oficial de Población de 2001). Esta complementariedad se muestra especialmente evidente en un territorio concreto que se localiza en la interrelación entre ambas, contemplado inicialmente por la planificación regional como corredor Ciudad RealPuertollano, espacio significativamente dinámico en base, principalmente, a la conexión por ferrocarril de alta velocidad y por autovía a partir de la cual se ha comenzando a reforzar la cohesión territorial.

Son estas infraestructuras de comunicación, principalmente la alta velocidad ferroviaria, que llega en 1992 en la línea Madrid-Sevilla y las autovías A-41 y A-43, de más reciente construcción dentro del eje Valencia-Lisboa, las que permiten, a ambas ciudades y a sus áreas funcionales, aprovechar las sinergias y colaborar en un territorio conjunto. Como resultado, se ha creado un eje de 
cierta vitalidad socioeconómica organizado en torno a los núcleos de Daimiel, Ciudad Real y Puertollano, que muy bien puede prolongarse hasta Manzanares alargando el corredor e incluyendo a otro de los asentamientos urbanos más importantes. Conexión reforzada por la existencia de una infraestructura de comunicación de gran relevancia pero corta vida, como es el aeropuerto Ciudad Real Central (en funcionamiento desde diciembre de 2008 hasta octubre de 2011), cuya eventual reapertura y óptimo funcionamiento serviría para complementar la oferta de comunicaciones y relanzar las dinámicas socioeconómicas de los municipios de esta zona.

No obstante, el factor de oportunidad generado por las nuevas infraestructuras es destacado, pero insuficiente en sí mismo, y más aún en el presente contexto. La ordenación del territorio bajo criterios de cohesión es una herramienta óptima que debe permitir, a través de la oportuna planificación, aprovechar estas ventajas y cumplir los objetivos de orden regional que se plantean. Esto es aún más importante en el entorno actual de recesión económica, donde la optimización de los recursos debe prevalecer sobre otras prioridades. No debe olvidarse, tampoco, que el análisis se ha realizado considerando el corredor Ciudad RealPuertollano como objeto de planificación territorial provisional (aunque, en caso de no ser objeto de la misma, este eje presenta autonomía propia), y que el citado aeropuerto debe incluirse, una vez activo, en la oferta de comunicaciones.

A pesar de todo, y para finalizar, señalamos que focalizar el esfuerzo planificador en áreas con gran capacidad estructurante puede dejar de lado importantes áreas que se quedarían, así, desconectadas de los sistemas generales. Es, por tanto, una labor importante en materia de planificación territorial la que debe hacerse para impedir la creciente polarización de nuestros espacios y el pleno aprovechamiento de las nuevas oportunidades que se han generado en las últimas décadas de crecimiento.

\section{Referencias bibliográficas}

AAlbU, Hallgeir (2004). «Europa policéntrica ¿̨utopía o realidad?». En: FARINÓs, Joaquín y Romero, Joan (eds.). Ordenación del Territorio y Desarrollo Territorial. Gijón: Trea, 145-170.

CAÑIZARES, María del Carmen (1999). «La red urbana». En: TAMAMES, Ramón y Heras, Raúl (eds.). Enciclopedia de Castilla-La Mancha. Vol. II. El espacio humano. Madrid: Edicsa, 77-95.

- (2001). El proceso de urbanización de la ciudad de Puertollano. Ciudad Real: Diputación Provincial de Ciudad Real.

- (2007). «Los cambios y las estrategias territoriales: Hacía una correcta ordenación del territorio en Castilla-La Mancha». Biblio 3W. Revista Bibliográfica de Geografia y Ciencias Sociales [en línea], XII (704), 10 de febrero de 2007. <http://www. ub.edu/geocrit/b3w-704.htm> [Consulta: 5 noviembre 2012].

- (2009). «Planificación Territorial e Infraestructuras de Transporte en Castilla-La Mancha: El Corredor Ciudad Real-Puertollano». En: CARAVACA, Inmaculada; SALINAS, Víctor y SILVA, Rocío (dirs.). Ciudades, culturas y fronteras en un mundo en cambio. Sevilla: Junta de Andalucía. Consejería de Obras Públicas y Transportes, 184-196. 
- (2010a). «Transformaciones territoriales y planificación en el Corredor Ciudad Real-Puertollano (Castilla-La Mancha)». En: Cornejo, C.; Morán, J. y PradA, J. (coords.). Ciudad, territorio y paisaje: Reflexiones para un debate multidisciplinar. Madrid: CERSA, 156-171.

- (2010b). «Transformaciones en el paisaje urbano-industrial de la ciudad de Puertollano (Ciudad Real)». En: Delgado, Carmen (ed.). Espacios y Paisajes Urbanos: Reflexionar sobre su presente para proyectar su futuro. Santander: AGE, Universidad de Oviedo, Universidad de Cantabria y Universidad del País Vasco, 156-166.

- (2012) «Cambios recientes en el paisaje urbano-industrial de la ciudad de Puertollano: Entre la supervivencia y la modernización». En DeLGaDo, Carmen (ed.). Ciudades y paisajes urbanos en el siglo XXI. Santander: Ediciones de Librería Estudio, 93-110.

Cebrí́n, Francisco (2007a). «La red urbana». En: Pillet, Félix (coord.). Geografía de Castilla-La Mancha. Ciudad Real: Almud, Ediciones de Castilla-La Mancha, 177-194.

- (2007b). «Transformaciones y ajustes ante las nuevas dinámicas urbanas: Manifestaciones en la organización del territorio de Castilla-La Mancha». Estudios Geográficos, 262, 7-32.

Cebriân, Francisco; García, Juan Antonio y Panadero, Miguel (2010). «Dinámicas y transformaciones recientes en los procesos de expansión territorial de las ciudades medias en Castilla-La Mancha». En: Delgado, Carmen (ed.). Espacios y paisajes urbanos: Reflexionar sobre su presente para proyectar su futuro. Santander: AGE, Universidad de Oviedo, Universidad de Cantabria y Universidad del País Vasco, 19-31.

Comisión de las Comunidades EuRopeas (2008). Libro Verde sobre la cohesión territorial. Convertir la diversidad territorial en punto fuerte, Bruselas (6-10-08). [en línea] < http://europa.eu/legislation_summaries/regional_policy/review_and_future/pr0002_es.htm> [Consulta: 4 marzo 2013].

Consejería de Ordenación del Territorio y Vivienda (2009a). Plan de Ordenación del Territorio "Estrategia Territorial de Castilla-La Mancha». Documento de Aprobación Inicial. Toledo: Junta de Comunidades de Castilla-La Mancha, Toledo (formato CD).

- (2009b). Plan de Ordenación del Territorio "Corredor Ciudad Real-Puertollano". Documento de Aprobación Inicial. Toledo: Junta de Comunidades de Castilla-La Mancha (formato CD).

CORONADO, José María et al. (2011). «Alta velocidad, integración metropolitana y proyectos territoriales: El caso de Ciudad Real y Puertollano». Urban, 9, 30-44.

FARINOS, J. (2001). «Políticas, territorio y geografía: La nueva visión del desarrollo territorial europeo». En: VV.AA. Actas del XVII Congreso Nacional de Geografia. Oviedo: AGE y Universidad de Oviedo, 307-311.

FundaCión DE los FerRoCARRILes Españoles (2011). Observatorio del Ferrocarril en España. Informe 2010 [en línea]. Documento de trabajo, diciembre de 2011. $<$ http://www.observatorioferrocarril.es/archivos/Ofe2010/ ObservatorioFerrocarril_2010.pdf> [Consulta: 5 noviembre 2012].

GARMENDIA, Maddi (2007). «Alta velocidad y cohesión territorial: El sistema urbano de Ciudad Real». En: Ribalaygua, Cecilia (ed.). Alta Velocidady Territorio. Ciudad Real: Universidad de Castilla-La Mancha, 93-113. Cuadernos de Ingeniería y Territorio, 10. 
Garmendia, Maddi; Ureña, José María y Coronado, José María (2011). "Cambios en la estructura territorial debidos a nuevas conexiones de alta velocidad en territorios aislados: la provincia de Ciudad Real en España». Eure: Revista Latinoamericana de Estudios Urbano Regionales, 37 (110), 89-115.

Green, Nick (2007). «Functional Polycentricity: A Formal Definition in Terms of Social Network Analysis». Urban Studies, 44 (11), 2077-2103.

Hall, Peter y Pain, Kathy (2006). The Polycentric Metropolis: Learning from Mega-City Regions in Europe. Londres: Earthscan.

Marmolejo, Carlos; Chica, Eduardo y Masip, Jaume (2011). "¿Hacia un sistema de metrópolis españolas policéntricas?: Evolución de la influencia de los subcentros en la distribución de la población». ACE. Arquitectura, Ciudad y Entorno [en línea], año 6 (18), febrero, 163-190. <http://www-cpsv.upc.es/ace/Articles_n18/ articles_PDF/ACE_18_SE_25.pdf> [Consulta: 5 noviembre 2012].

MartíneZ, Héctor Samuel (2010). La estructura del transporte en Castilla-La Mancha y sus implicaciones territoriales. Toledo: Consejo Económico y Social.

- (2012). «La accesibilidad regional y el efecto territorial de las infraestructuras de transporte: Aplicación en Castilla-La Mancha». Boletín de la Asociación de Geógrafos Españoles, 59, 79-103.

MéndeZ, Ricardo y PRADA, José (2010). "Ciudades y desarrollo territorial en Castilla-La Mancha». En: Cebriân, Francisco; Pillet, Félix y CARPIO, José (eds.). Las escalas de la Geografía: del mundo al lugar: Homenaje al profesor Miguel Panadero Moya. Cuenca: Ediciones de la Universidad de Castilla-La Mancha, 153-174.

MEnÉndeZ, José María (2004). «La Mancha, tierra de paso: Los itinerarios en el eje de comunicación norte-sur». En: Pedregal, José María (dir.). Obras públicas en Castilla-La Mancha. Madrid: Ministerio de Fomento y Junta de Comunidades de Castilla-La Mancha, 103-124.

Menéndez, José María; Coronado, José María y Rivas, Ana (2002). El AVE en Ciudad Real y Puertollano. Ciudad Real: Universidad de Castilla-La Mancha. Cuadernos Ingeniería y Territorio, 2.

Panadero, Miguel; García, Carmen y Cañizares, María del Carmen (1999). "Continuidad y cambio en la estructura socioeconómica de las ciudades de Castilla-La Mancha». En: VV.AA. Professor Joan Vilá Valentí: El seu mestratge en la geografía universitaria. Barcelona: Universidad de Barcelona, 1153-1173.

Panadero, Miguel y Pillet, Félix (1999). «Castilla-La Mancha». En: García, José María y SoTElo, José Antonio (coords.). La España de las autonomías. Madrid: Síntesis, 291-330.

Pillet, Félix (1984). Geografía Urbana de Ciudad Real. Madrid: Akal.

- (2005). "Ciudad Real: cambios en el espacio público y en la vida colectiva con la llegada del AVE a la ciudad». En: VV.AA. La ciudad y el miedo. Girona: AGE y Universitat de Girona, 325-334.

- (2012). Planificación territorial: Propiedad y valoración catastral (España 17502010). Madrid: Biblioteca Nueva.

Pillet, Félix et al. (2007). «Fuentes para la aplicación de la Estrategia Territorial Europea en Castilla-La Mancha». Estudios Geográficos, 68 (263), 627-651.

- (2010). «El policentrismo en Castilla-La Mancha y su análisis a partir de la población vinculada y el crecimiento demográfico». Scripta Nova: Revista Electrónica de Geografía y Ciencias Sociales [en línea], XIV (321), 20 de abril de 2010. <http:// www.ub.edu/geocrit/sn/sn-321.htm> [Consulta: 5 noviembre 2012]. 
Plaza, Julio; Martínez, Héctor Samuel y Gosálvez, Rafael Ubaldo (2010). «La ordenación del territorio en Castilla-La Mancha: estado de la cuestión y estudio de casos». Cuadernos Geográficos, 47, 493-522.

PONS, Juan José et al. (ed.) (2009). Territorio y movilidad interior de la población Española. Pamplona: EUNSA.

RocA, Josep; MoIX, Montserrat y Arellano, Blanca (2012). «El Sistema Urbano en España». Scripta Nova: Revista Electrónica de Geografía y Ciencias Sociales [en línea], XVI (396), 20 de marzo de 2012. <http://www.ub.edu/geocrit/sn/sn-396.htm> [Consulta: 20 diciembre 2012].

RODRÍGUEZ, María de los Ángeles (2012). La nueva realidad urbana y territorial de Ciudad Real (1980-2010). Ciudad Real: Instituto de Estudios Manchegos.

SANTOS, Jesús Francisco y MARTíneZ, Héctor Samuel (2010). «Polígonos industriales en torno a la nueva autovía A-43 entre Ciudad Real y Manzanares. Situación, diagnóstico y perspectivas». En: VV.AA. Actas de las IV Jornadas del Grupo de Geografía Económica [en línea]. León, AGE y Universidad de León. <http://age. ieg.csic.es/geconomica/IVJornadasGGELeon/Comunicaciones\%20Jornadas\%20 de\%20Leon/I\%20Ponencia/Santos\%20y\%20Martinez.pdf> [Consulta: 5 noviembre 2012].

Solís, Eloy (2011). Del área metropolitana hacia la región urbana policéntrica madrileña: Cambio de escala, estructura y articulación territorial. Tesis doctoral. Madrid: Universidad Complutense de Madrid.

UE (1999). Estrategia Territorial Europea. Bruselas, Luxemburgo.

- (2007). Agenda Territorial Europea. Hacia una Europa más competitiva y sostenible de regiones diversas. Disponible en [en línea]. <http://www.upv.es/contenidos/ CAMUNISO/info/U0556578.pdf> [Consulta: 4 marzo 2013].

- (2011). Agenda Territorial de la Unión Europea 2020: Hacia una Europa integradora, inteligente y sostenible de regiones diversas [en línea]. <http://www.marm.es/es/ desarrollo-rural/temas/desarrollo-territorial/ Agenda_Territorial_Europea_2020_ adoptada_en_G\%C3\%B6d\%C3\%B611\%C3\%B6_en_2011_tcm7-172182.doc> [Consulta: 5 noviembre 2012].

VinUESA, J. (2005). «De la población de hecho a la población vinculada». Cuadernos Geográficos, 36, 79-90.

\section{Páginas web}

INE: www.ine.es [Consulta: 6 noviembre 2012].

Red Polynet: www.polynet.org.uk [Consulta: 3 enero 2013].

Servicio de Estadística de CASTILla-La MANCHA: http://www.ies.jccm.es/ [Consulta: 6 noviembre 2012]. 
